\section{Developing Large High-Resolution Display Visualizations of High-Fidelity Terrain Data}

\author{
Haeyong Chung \\ e-mail: chungh@vt.edu \\ Chris North \\ e-mail: north@cs.vt.edu \\ Department of Computer Science, \\ Virginia Tech, \\ Blacksburg, VA 24060
}

\author{
John Ferris \\ Department of Mechanical Engineering, \\ Virginia Tech, \\ Blacksburg, VA 24060 \\ e-mail: jbferris@vt.edu
}

The vehicle terrain measurement system (VTMS) allows highly detailed terrain modeling and vehicle simulations. Visualization of large-scale terrain datasets taken from VTMS provides better insights into the characteristics of the pavement or road surface. However, the resolution of these terrain datasets greatly exceeds the capability of traditional graphics displays and computer systems. Large high-resolution displays (LHRDs) enable visualization of large-scale VTMS datasets with high resolution, large physical size, scalable rendering performance, advanced interaction methods, and collaboration. This paper investigates beneficial factors, implementation issues, and case study applications of LHRDs for visualizing large, high-fidelity, terrain datasets from VTMS. Two prototype visualizations are designed and evaluated with automotive and pavement engineers to demonstrate effectiveness of LHRDs for multiscale tasks that involve understanding pavement surface details within the overall context of the terrain. [DOI: $10.1115 / 1.4024656]$

\section{Introduction}

Advances in modern 3D laser measurement systems, GPS, and various sensor technologies make it possible to obtain detailed high-fidelity terrain measurements. For example, the VTMS scans pavement and other road surfaces with millimeter precision over large areas and long driving distances [1]. These terrain measurements are of particular interest to automotive and pavement engineers who perform vehicle simulations and pavement evaluations. Such data enable detailed analysis and high-fidelity simulation of interactions between vehicle systems and terrain, to predict behaviors and validate vehicle designs. Multiscale terrain data that are both detailed and large in extent are very useful for monitoring pavement health and planning road repair $[2,3]$.

Simultaneously, advances in LHRD systems have made it possible to visualize large multiscale data [4] (for example, see Fig. 1). LHRD significantly increases visual scalability over standard small displays with its large field of view and high resolution [5], enabling visualizations to display a large amount of detailed data. Such displays have shown significant userperformance advantages in a variety of visual analytics and com-

Contributed by the Computers and Information Division of ASME for publication in the Journal of Computing and Information Science in Engineering. Manuscript received June 30, 2011; final manuscript received March 26, 2013; published online July 22, 2013. Editor: Bahram Ravani. plex information work tasks that involve a large amount of data in other data domains [6-8].

In this paper, we propose the coupling of high-fidelity terrain measurement systems with large high-resolution display systems to support detailed visual analysis of terrain by automotive and pavement engineers. The main purpose of this paper is to demonstrate and accommodate actual pavement analyses utilizing both VTMS and LHRD. Our basic hypothesis is that the combination of both systems will enable engineers to better understand, validate, and compare high-fidelity terrain datasets, in conjunction with vehicle simulations and pavement engineering, in a more interactive and collaborative manner.

To that end, we design, develop, and evaluate two prototype visualizations of high-fidelity terrain data on an LHRD to support various pavement analysis tasks. First, because developing software for LHRD visualizations requires complex distributed multipipe graphics architectures for real-time interactive performance, we compare architectural tradeoffs in support of our prototypes in the context of high-fidelity terrain data to help developers' select suitable technologies for their specific visualizations of largescale pavement modeling data for large displays. Second, we describe the design and implementation of two prototypes for true-to-life terrain visualization and multiple-view data visualization. Third, we test these prototypes in two case studies with automotive and pavement engineers to evaluate how these visualizations can enable engineers to examine pavement details while maintaining the context of the global pavement condition and vehicle responses.

\section{Background}

Vehicle Terrain Measurement System. Terrain measurement systems began by recording a 2D vector of heights along a longitudinal distance traveled to examine pavement roughness [9]. Recent optical and computational advances have produced pavement 3D profiling scanners [10] which measure a full vehicle lane width (about $4 \mathrm{~m}$ ), so that 3D surfaces, rather than 2D vectors, are recorded. More recently, the VTMS has been developed by the authors, as shown in Fig. 2 [1]. The VTMS is composed of two subsystems: (1) a scanning laser which directly measures the digital terrain models at the rear of the vehicle and (2) an integrated system of an Inertial Navigation System (INS) and accelerometers which provide the position and orientation information of the scanning laser in space. The system is capable of simultaneously scanning a four-meter-wide topology of the terrain while simultaneously tracking the position, orientation, and speed of the vehicle. The VTMS creates terrain measurements with transverse spacing of five millimeters and a vertical resolution of one millimeter. Figure 3 shows the resolution achieved through VTMS.

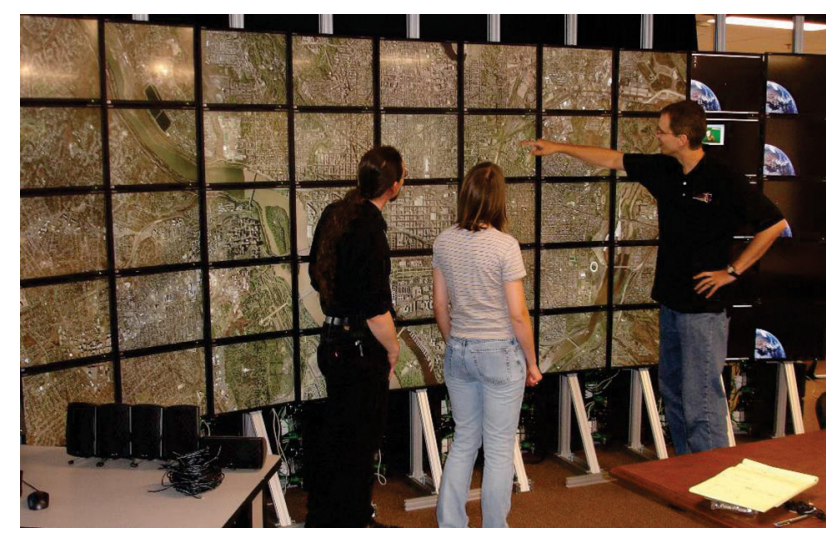

Fig. 1 Large, high-resolution display, arranged in a $5 \times 10$ matrix of 20.1 in. flat panel LCD monitors powered by 25 PC nodes $(5 \times 10 \times 1600 \times 1200=96,000,000$ pixels in total $)$ 


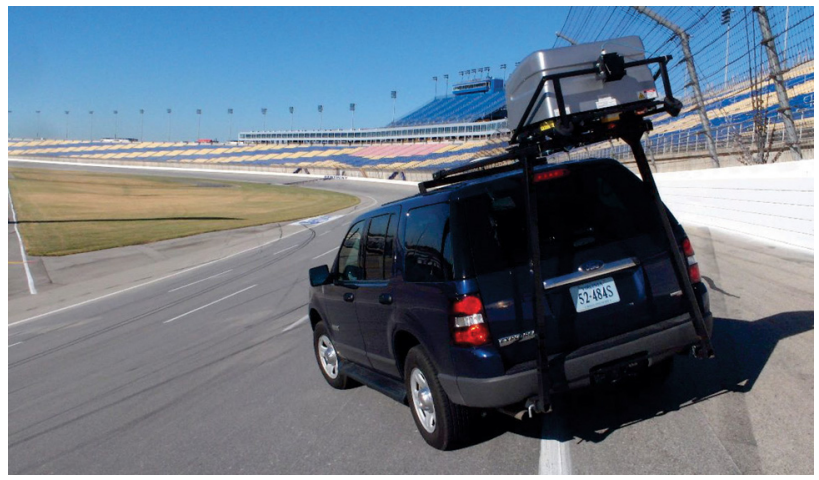

Fig. 2 VTMS

Use of these 3D data allows validation of vehicle models, ride quality [11], tuning of chassis components [12], and vehicle mobility and durability studies [13]. During the vehicle design and development process, vehicle models are simulated traveling over specific events typically found on a durability test road at a vehicle manufacturer's proving ground or public roads and off-road trails. As a vehicle traverses the terrain, it is excited by the roughness of the road, resulting from cracks, bumps, and dips. This excitation causes the vehicle to respond by developing loads, moments, deflections, deformations, etc. Throughout the development cycle of a ground vehicle, it is critical to accurately predict the loads that a vehicle will experience from the terrain.

Large High-Resolution Displays. Since the early 1990s, LHRDs have been increasingly used for large-scale visualization [14]. Prior research by Yost et al. demonstrated that user performance in various large-scale visualization tasks is improved by increased simultaneous access to information, not only through LHRD's physically large size but also their high-resolution [15]. If a multitiled display is driven by a single computer system, it is almost impossible to avoid decreasing processing power and the usage of system resources with high bus bandwidth, since the multiple displays share the single computer's hardware resources including CPU, GPU, memory, and HDD. Therefore, tiled displays driven by a commodity PC clusters are recognized as an efficient and cost-effective solution for implementing LHRDs [16].

Despite a recent dramatic advance in size and resolution in display technologies, the scalability of single monitor displays is still limited by engineering and manufacturing constraints on dot per inch (DPI) and large physical size. A higher DPI increases the quantity of detail and information that can be displayed through a greater number of pixels per inch. The DPI affects the usability of applications on LHRDs, such as how users can exploit the full range of physical navigation and all the benefits of the display space [5].

Two types of tiled arrays, LCD monitors and projectors, are typically used to provide more scalability in both the size and resolution. However, a tradeoff exists between the two types of displays. Projection-based powerwall have the advantages of being seamlessly tiled and easily scaled-up in the size; however, they contain less pixel density and achieve less brightness than LCD-based tiled displays, and they require calibration processes such as adjusting differences of brightness and colors uniformly across display tiles. There are, of course, solutions for tiling different projectors together, but it remains difficult to achieve the same pixel density and clarity as with LCD tiled displays.

In this paper, we refer to the large tiled display of LCD monitors as LHRDs because the LCD-based tiled display provides the highest DPI relative to physical size [15] among the different types of the tiled displays. Our LHRD system in Fig. 1 is developed with off-the-shelf components, including 50 affordable LCD flat panel monitors, graphics cards, and a PC cluster of 25 inexpensive desktop PCs. In this configuration, each one or two display tiles is assigned to a single PC node in the cluster, and every node takes care of a part of the rendering tasks and collaborates with other nodes to create a large, single, coherent image across multiple display tiles.

\section{Motivation}

Users often meet the following problems when visualizing and navigating a large, high-fidelity terrain. These problems became the main motivations to use LHRD for visualizing a large dataset from VTMS in our study.

- Display resolution. The high-fidelity visualization supported by current normal desktop displays is often limited by both pixel resolution and physical size, since the resolution and size of high-fidelity terrain data from VTMS easily exceed the display resolution and performance even on today's high-end displays and computer systems.
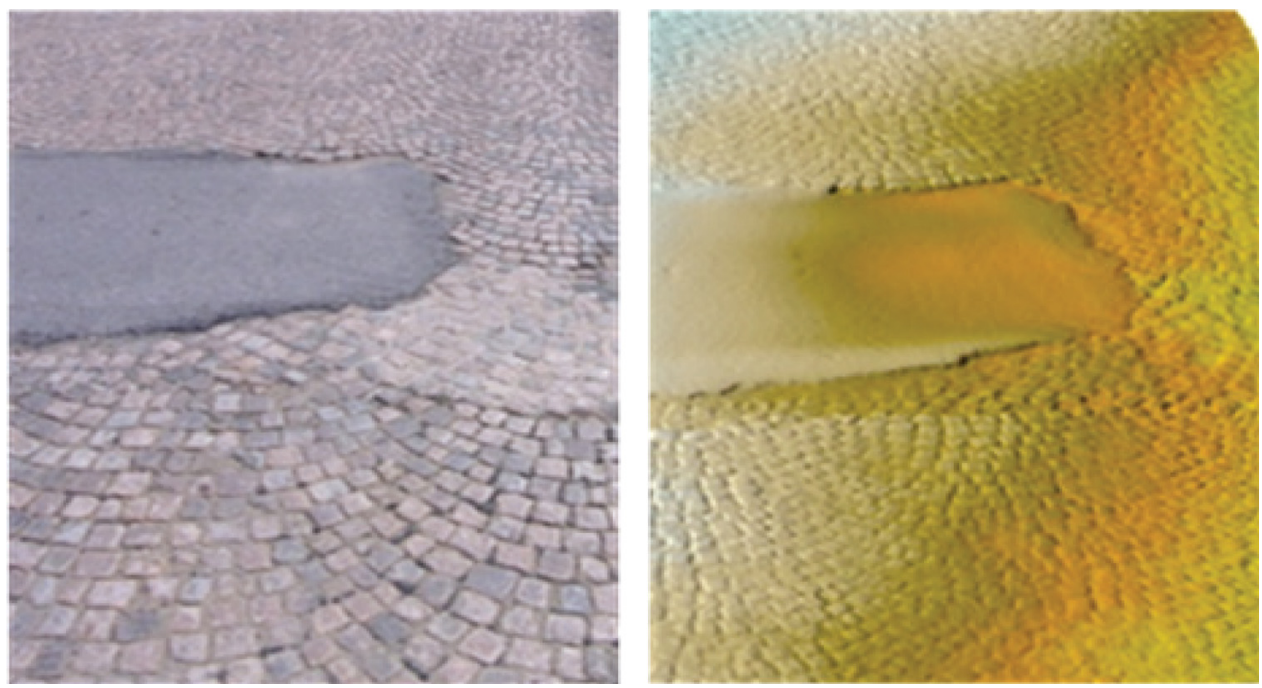

Fig. 3 Actual terrain and 3D terrain visualization. The left image is a photo of the actual terrain and the right image is a 3D rendering that was produced from the corresponding dataset measured with the VTMS. 
- System performance. VTMS generates a set of "XYZ" values, or point clouds, not directly useable in 3D terrain visualization. Therefore, the point cloud data are converted into a polygon mesh using various $3 \mathrm{D}$ reconstruction techniques [17]. It is very difficult for users to visualize the entire terrain surface without encountering bottlenecks and sacrificing precision.

- Interactions. Interaction is essential to examine large-scale terrain data from VTMS. However, virtual navigation, such as mouse control, panning, and zooming commonly used in small display applications, is also required to frequently change the viewpoint of terrain models. This has a disorienting effect on users performing spatial analysis tasks [18].

Cluster-based LHRDs can provide effective solutions for solving these problems. The following benefits will accrue when LHRD systems are applied to the above-mentioned problems for visualizing VTMS data.

- Supporting high-resolution terrain visualization. VTMS can resolve terrain features on a wide range of scales, including less than one millimeter texture measurement. It is crucial that the terrain visualization for the VTMS data does not hide detail within the terrain surfaces and does not provide false information to users. LHRD visualizations make full use of a wide field of view and greater DPI, and they enable users to see a large amount of terrain information by reconstructing a multiscale terrain in a nearly physical true-to-life size. They also provide broader contextual overview of the entire terrain condition. The larger physical size and resolution of LHRDs can improve the user's experience and performance on 3D spatial tasks when users interact with virtual environments [19].

- Advanced interactivity and physical navigation. LHRD visualizations afford the use of advanced interaction devices and physical navigation that effectively exploit human spatial senses. For example, to detect an interesting feature on the terrain, users can directly manipulate terrain models by using a wide range of natural input devices such as pointing gestures, touch-screens (see Fig. 4), 6-degree-of-freedom (6DOF) tracker, head tracking interaction, a 3D mouse, etc. With a great deal of open space in front of the LHRD, the display affords the use of physical navigations such as turning the head, leaning the torso, or walking [18].

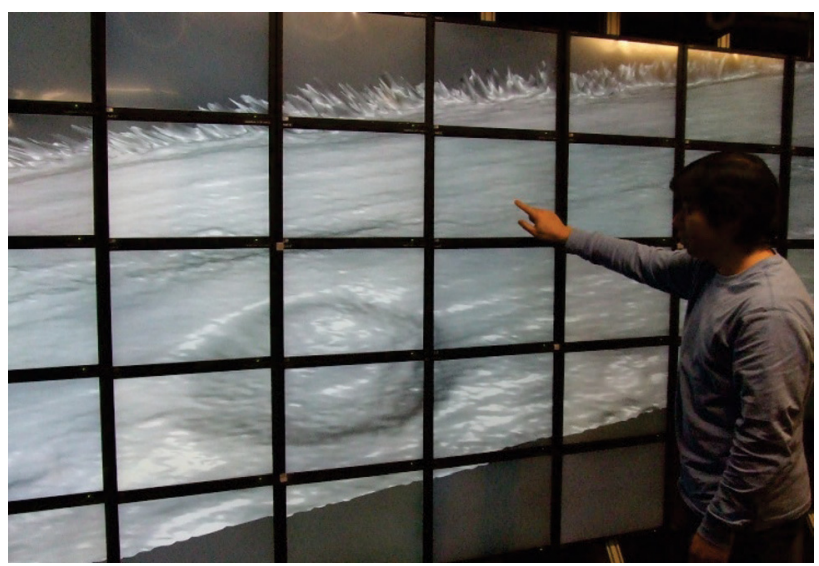

Fig. 4 Manipulating a 3D terrain model with a touch-screen interface. A user is inspecting a large pothole on a road.
- Supporting collaboration. LHRD's larger peripheral views with higher resolution offer excellent benefits to group collaboration and discussion of data sets [15]. LHRD supports multiple user inputs and views. It allows a group of people to watch and work in front of the display at the same time, and the 3D view of terrain can be controlled by multiple users at the same time. LHRD inherently supports collaboration work in visual analyses of terrain surface and simulation data from its larger field of views. It provides co-located engineers with multiple, related views of terrain profiles and allows multiple users to work and discuss within the shared display space simultaneously.

- New virtual terrain environment. LHRD visualization provides different types of virtual terrain environments from alternative display technologies such as head-mounted displays, cave automatic virtual environment (CAVE), and large projection screens. These alternative displays provide the similar physical size properties to the LHRD, but either they lack the ability to display the same density of highfidelity data from VTMS due to the lower-resolution or the perspective and navigation techniques of a single user are preferred (i.e., perspective translation, panning or zooming by a single user). These technologies aim to immerse the users in a virtual world and hide our physical world; however, LHRDs benefit from the large physical space in front of the display, creating a new terrain environment that combines our physical and virtual space [18]. Use of the physical space can offer greater opportunities to employ our visual perception and spatial abilities for visualization tasks.

\section{Developmental Choices and Implementation}

In this section, we discuss different approaches affecting the performance and quality of terrain rendering on LHRDs. A successful LHRD visualization has a strong relationship to the selection of appropriate task and data distribution architectures and parallel rendering methods.

Task Distribution Architectures. In cluster-based multitile displays, the most important issues are how graphics rendering tasks and large-scale terrain data are distributed, maintained, and executed in networked nodes. The greatest performance overhead in cluster-based LHRD systems occurs at the network communication level among the nodes [20]. The task and data distribution model is more critical for terrain visualization, since the amount of VTMS data to be transmitted is extremely large. In general, data distribution architectures are classified in two ways, depending on which type of rendering tasks and data are transmitted among the nodes and upon how an application's tasks are distributed across nodes in the cluster: Master-slave or client-server $[16,21,22]$. The most important developmental consideration was to provide scalable rendering performance (more than 15 frames/ s) through parallel rendering by executing a part of the application or rendering tasks in parallel across cluster nodes. Merely increasing resolution of the visualization is not enough for our applications that will be described in detail later. Thus, we use the clientserver model for our terrain visualization, which separates the rendering tasks from the core application, performs the rendering tasks in each display server node in parallel and the application tasks are performed in a single head node, as shown in Fig. 5(b). This model does not keep separate copies of the large VTMS data in every node. Whereas, in the master-slave model, the same copies of the large VTMS data and applications must be maintained and iterated with different view parameters in each node of the cluster (Fig. 5(a)). While the master-slave model enables to minimize transmissions of rendering data among the nodes in the cluster, its utilization of distributed graphics hardware of the LHRD 
(a)

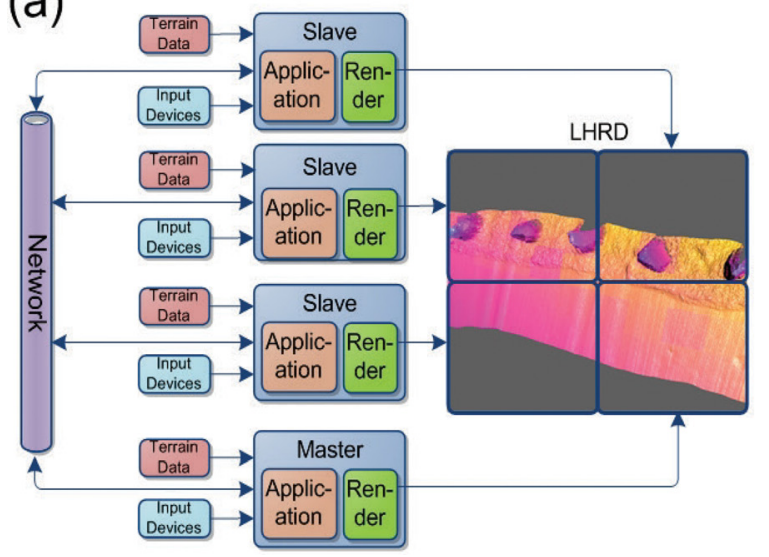

(b)

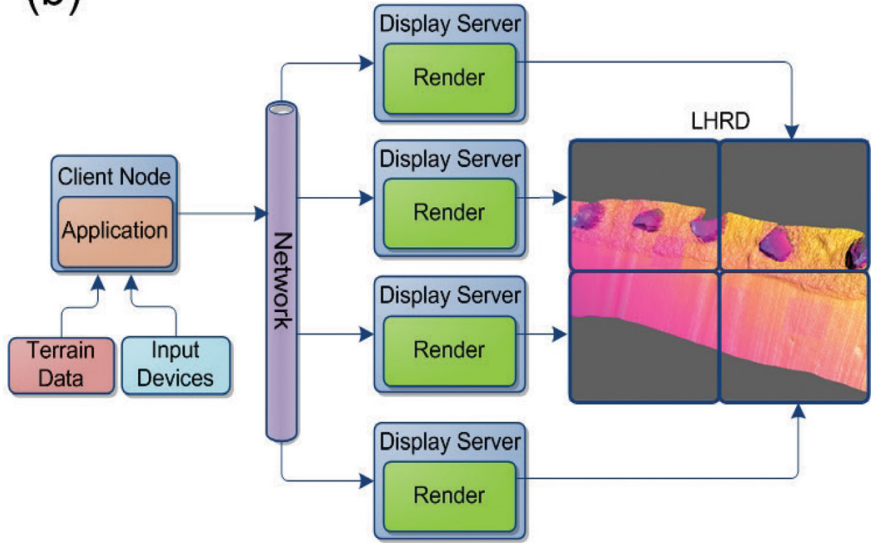

Fig. 5 Two different data distribution architectures for terrain data on LHRDs. (a) Master-slave data distribution architecture. The master redistributes application state information collected from the slaves, such as user input, timer, random number generation, system calls, etc. in order to synchronize application states. (b) Client-server data distribution architecture. The terrain data and input devices are accessed by only the client node.

cluster is less efficient for the VTMS data due to these characteristics.

Parallel Rendering Algorithms. With parallel rendering algorithms, the entire rendering task is divided into small parts, and those parts are rendered at associated nodes. Molnar proposed parallel rendering algorithms based on where the sorting takes places in the rendering pipeline [23]. For parallel rendering, we used the sort-first method because the visualization requires interactive terrain manipulations and lower network bandwidth (Fig. 6(left)). Since LHRDs are inherently partitioned with the display tiles, this algorithm is regarded as the most suitable sorting algorithm to drive multiple-tile displays [24]. While the sort-last method enables to divide the terrain model more evenly, this method requires reading back the rendered image pixels in each of the nodes in order to compose them into the final terrain on the large display (Fig. 6(right)) and it causes serious network overhead as the size of VTMS data increases [25]. Therefore, sort-last is more appropriate for static visualization in order to visualize an extremely large terrain data set [26].

Maintaining the Large Terrain Model. In order to organize a large-scale terrain data set efficiently and to optimize rendering performance in each node, we use a 3D kD-tree [27]. We spatially organize the large-sized VTMS data into a 3D kD-tree. When starting the visualization, the application preprocesses the terrain model into the $\mathrm{kD}$-tree. A data range is spatially assigned to each $\mathrm{kD}$-tree node. This facilitates view frustum culling in each display tile by simply testing to determine whether a partial terrain model is inside each node's view frustum. Therefore, the $\mathrm{kD}$-tree approach helps each display node to save its graphics hardware resource and improves the overall performance of the LHRD cluster.

In addition, we also use openGL's display lists. Terrain visualization primarily requires frequent changes to the view transformation matrix, rather than changing actual geometry. Thus, we use OPENGL display lists to repeatedly reuse a group of rendering commands that have been cached on the display server node. Once a display list is compiled, the client does not need to retransmit the rendering data and functions to every render node. This approach reduces data transmission between the client and the multiple renderer nodes.

Software Toolkit. All prototype visualizations are implemented with Equalizer, an OPENGL-based parallel rendering system. Equalizer is cross-platform compatible and available for
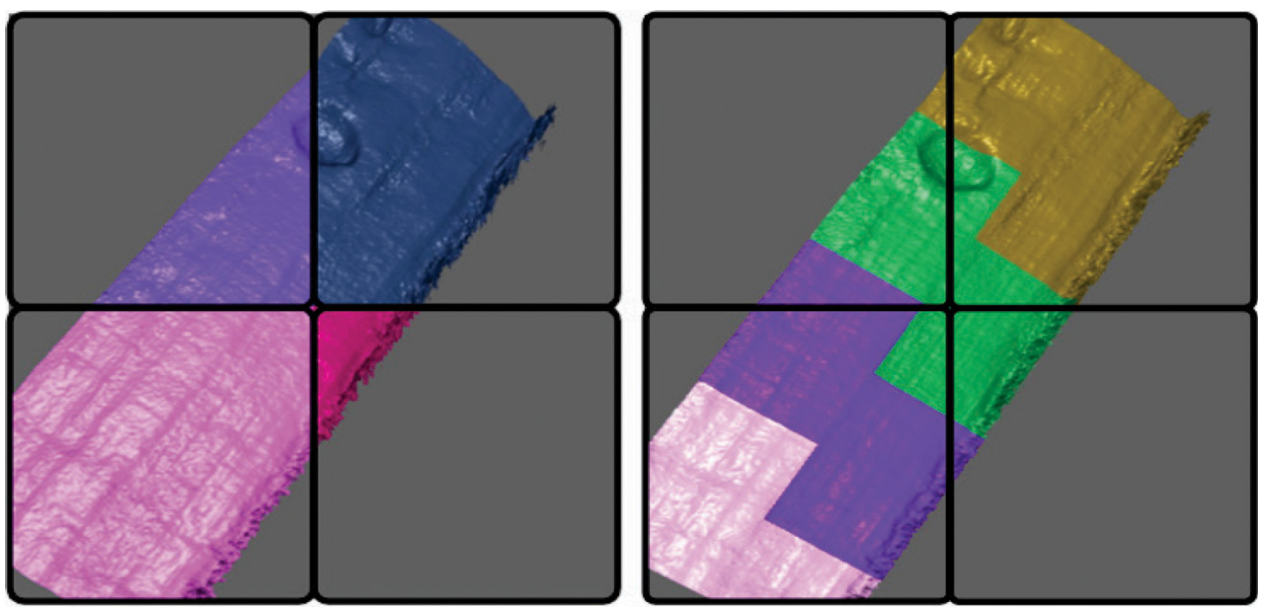

Fig. 6 Left: Sort-first rendering of VTMS data. Black lines represent display tile borders. The entire terrain model is divided by display tiles, and then display nodes (represented by different colors) render the corresponding parts of the terrain model in parallel. Right: Sort-last rendering. The terrain model is more evenly divided. 
Linux, Windows XP, and Mac OS X. It provides scalable rendering performance for cluster-based LHRDs with improved parallelism by executing a part of the application code in parallel across multiple cluster nodes [28]. For realistic shading effects on the surface, we also used GLSL (OPENGL shading language).

\section{Visualization Prototypes}

We designed and implemented two visualization prototypes of the VTMS data to demonstrate effectiveness of LHRD for tasks that involve understanding pavement surface details within the overall context of the terrain.

True-to-Life Terrain Visualization. Our first LHRD prototype helps with identifying terrain topology characteristics. Specifically, in the case of paving a road, these renderings could be used to identify uneven surfaces and low or high points in the pavement, which need to be filled in or smoothed prior to the pavement setting (see Fig. 7). This prototype is capable of visualizing long stretches of terrain (greater than $50 \mathrm{~m}$ ), tracks, highways, and rough city streets. It allows users to observe the terrain surface at almost true-to-life scales and this is useful to inspect the entire terrain condition and distresses. In an actual on-site inspection setting, road surface defects are often concealed by inconsistent illumination effects resulting from the location of the sun, shadows cast by large buildings, or poor weather conditions. Whereas, the combination of high-fidelity terrain measurement and LHRD visualization enables pavement engineers to easily detect and identify these surface defects clearly through its highresolution and wide field of view, because users can choose the type of visual representations such as color ramps for encoding terrain elevation and text annotations. For interaction, users can zoom, pan, and rotate true-to-life size high-fidelity terrain models using a touch-screen or 6-DOF interface. Physical navigation can be efficiently exploited when analyzing the terrain data on the LHRD.

Multiview Data Visualization. The second type of LHRD visualization is based on a tabular layout "multiview" visualization facilitating evaluation of different states or parameters of a terrain
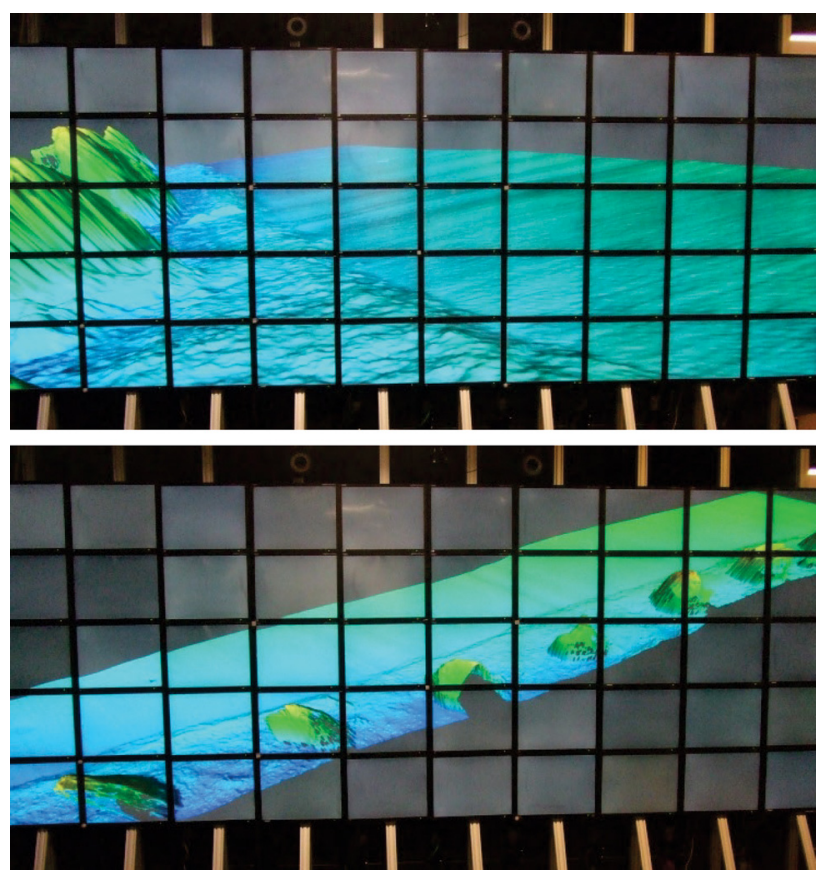

Fig. 7 Multiscale terrain visualization prototypes. Color ramp represents elevation of terrain. For example, red is the highest point and blue is the lowest.

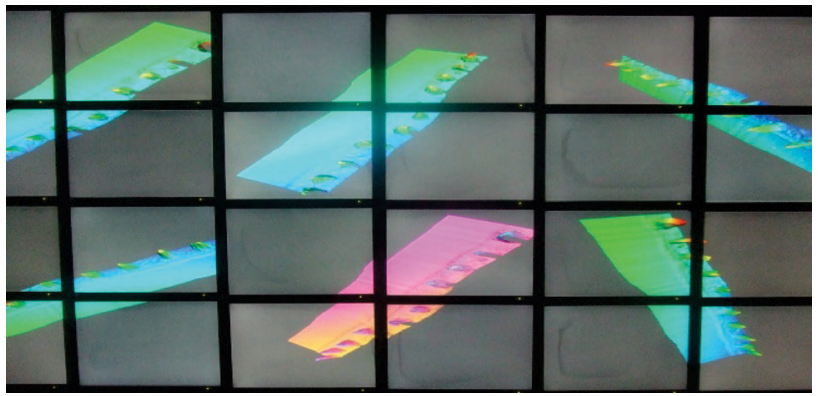

Fig. 8 Multiview visualization prototype for comparing performance of different terrain uniform grid spacing methods for VTMS data. Each model can be navigated independently or in coordination

dataset or simulation result simultaneously. This prototype visualization is inspired by NASA's Hyperwall [29]. This prototype is specially designed for exploring multidimensional datasets. The approach is different from typical LHRD visualization applications that present a single coherent image across the entire display.

This visualization prototype displays multiple related terrain representations, such as different (time) steps, different parameters, or different interpolation methods, similar to spreadsheetstyle approaches to multidimensional data visualization [30]. In this prototype, each display tile or some set of display tiles shows separate but related data. It enables users to compare different simulation results, multiple datasets, or a dataset using a different parameter. For example, we can evaluate and compare terrain models interpolated by different uniform grid spacing methods with different color codings simultaneously on the LHRD (Fig. 8). Also, a very long pavement segment can be visualized across multiple rows, while still maintaining the details and context of the entire pavement (Fig. 9).

\section{Use Cases}

We conducted two use cases of engineering applications for LHRD terrain visualization. The goals for the two use cases were to identify problems with existing user tasks and to determine whether a combination of VTMS and LHRDs can accommodate pavement analysis tasks and simulations for large-sized terrain datasets. Four different datasets were collected by VTMS, and our LHRD prototypes were used to support two different usage scenarios related to pavement evaluation and simulation. Each volunteer user carried out the terrain data inspection and analysis with our visualization prototypes on the LHRD system shown in Fig. 1 for an hour and a half. All datasets were collected by VTMS using a section of road located in Angler's Park, Danville, VA and the Smart Road at the Virginia Tech Transportation Institute. The author recorded and took notes regarding salient points or

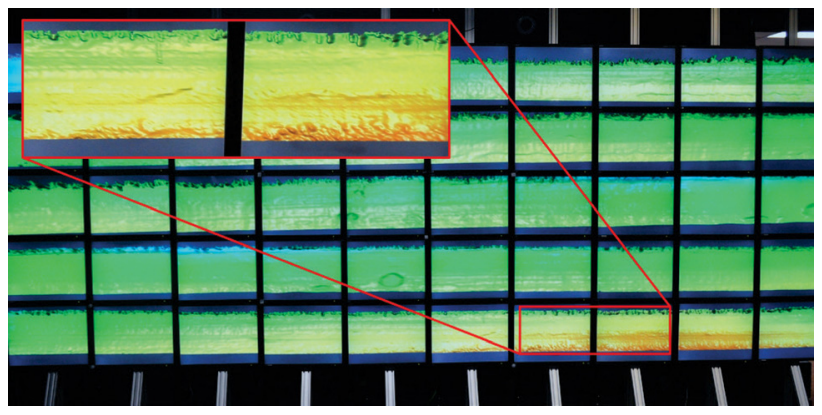

Fig. 9 Multiview visualization of very long pavement models. Each row displays a portion of the road length, and every row is connected to recreate the entire road section. 
observations during the sessions. A debriefing followed the session in which the author interviewed the users to elicit further observations about their experience in performing tasks using the visualization prototypes.

Multiscale Pavement Distress Evaluation. Road pavement distortions should be inspected and repaired properly to monitor and maintain the health, safety, and potential ride quality of roads. The main task of the first case study application is to determine properly the current condition of a pavement including cracking, fatiguing, distortion, and disintegration. Two senior pavement engineers who had previous in situ and image inspection experience conducted the collaborative tasks using the multiview visualization of very long pavement models (Fig. 9).

For actual pavement inspections, pavement inspectors mainly depend on visual inspection to determine necessary repairs [31] Based on the type and severity of the distress, they employ an objective rating system in order to identify the pavement condition, called pavement condition index (PCI) [32]. Generally, the pavement engineer needs to inspect a large number of sample images individually to calculate the PCI. Each sample covers the width of the pavement $(12 \mathrm{ft}, 3.7 \mathrm{~m})$ while the length is typically shorter (approximately $10 \mathrm{ft}, 3 \mathrm{~m}$ ) in $1024 \times 1300$ resolution at 1:200 scale. If the road section is very large, this inspection and PCI rating process can become very tedious and time-consuming, since users are required to load and inspect separate image sections iteratively.

Using the LHRD, the pavement engineers in our case study could see an entire road section that consisted of more than 20 sub units (the total length was greater than $60 \mathrm{~m}$ ). To accomplish the pavement condition rating for this section, both engineers frequently took advantage of physical navigation to inspect distress throughout the entire pavement evaluation. For example, both inspectors first stepped back from the display to find some interesting distresses on the entire section and then they stepped forward, toward the pavement units that had cracks, in order to see the cracks in detail. They also walked back and forth across the display between pavement units often. During the entire use case study, they repeated these processes often to inspect all of the pavement defects. When zoomed into the centimeter or millimeter level, the engineers appreciated that the large display provided context through physical navigation, enabling them to easily compare different distresses and to determine the reasons for cracking. The two users rated adjacent sample units of pavement in parallel, and they frequently discussed types and causes of the cracks. They commented that the LHRD was very helpful for the inspection task because it enabled them to easily see the full extent and detail of a crack at the same time. These results indicate that this user task and these experts' cognitive reasoning processes are inherently multiscale in nature, and that the LHRD appropriately afforded that multiscale process.

Data Editing for Vehicle Chassis Design. The goal of this case study application was to illuminate usability issues and process support for the task of verifying and editing vehicle simulation data using LHRD visualizations. Vehicle manufacturers use VTMS data for vehicle durability simulation and to inform the design process of vehicle chassis. For these purposes, the terrain data are used as input excitations for vehicle durability and dynamic simulations. Chassis engineers often need to inspect terrain surfaces with various excitation points (speed bumps, potholes, cracks, etc.), allowing them to see how the vehicle interacts with such terrain. These data are important factors for vehicle design, since they represent severe customer usage.

For accuracy of simulation results and design, it is essential to generate error-free surface data. Due to various software and hardware limitations, manual verification and editing of large datasets is required. Therefore, the terrain surface data must be visually inspected and corrected before they are used in simulations. These editing tasks involve eliminating ambiguities caused by laser error and removing impurities which may have been on the road surface.

In this case study, five engineers who regularly perform such tasks used our LHRD visualization prototypes (both multiview visualization and true-to-life visualization) to analyze new datasets. They used the following task procedure to create and edit the data. The pavement surface was first scanned using the VTMS system, which collects laser data, GPS and IMU data, and accelerometer data. These different types of data were then computationally processed together based on their timestamps to remove errors caused by vehicular body motions and dynamic changes in elevation, such as deep cracks in the road. However, occasionally, irregularities remain in the surface data. LHRD visualization of the data then played a crucial role in refining the data and the processing algorithms, because it enabled the engineers to easily see and correct for the different or abnormal interactions between the terrains and vehicles. Correction was accomplished through the modification and/or removal of data points in the source data file.

In our postsession interview, the engineers reported the following advantages of using the LHRD for their editing tasks. First, the high pixel density and large display size helped them to quickly and accurately pinpoint the location of ambiguities. They could easily distinguish data errors from normal events occurring on the road surface, and then remove them. For example, relatively small ambiguities caused by leaves or dirt or something laying on the road surface appeared clearly on the LHRD, and thus the display was very helpful to the process of selecting and removing those minute irregularities. Second, the users frequently performed panning and zooming interesting points with much less effort using physical navigation. All users preferred physical navigation for inspecting the data and performing the task, even though they could also navigate the data virtually using the touchscreen. Third, the users could effectively remove all of the errors in the data in one session, since it enabled them to render a much longer series of data at the same time, instead of tediously repeatedly loading, removing, and regridding separate small parts of the entire terrain. Therefore, the users did not need to constantly open and close short lengths terrain data to differentiate all of the errors on the surface as they were previously accustomed. These identified advantages indicate that benefits of LHRDs for visual search tasks that were previously identified in laboratory tasks [18] also carry over into these real world anomaly detection tasks.

\section{Discussion}

Bezels between display tiles on the LHRD can cause visual discontinuities for large pavement sections, even though the bezel width is small (around 0.75 in. between each pair of 20-in. monitors). In the case of the pavement distress evaluation, when a long pavement crack is split across multiple display tiles, there are some spatial distortions. Sometimes it appeared to the users that large cracks that crossed a bezel looked wider than actual. These spatial distortions could cause users to misinterpret the magnitude of the pavement distress. However, as shown by Tan and Czerwinski [33] and Bi et al. [34], bezels do not appear to affect users' overall performance on visual search time nor do they impact error rates. As reported by Robertson et al. [35], the bezels can provide both opportunities and problems. In our case study, the engineers simply used the touch-screen to interactively pan the image slightly to the side to move the point of interest away from the bezel and view it without local distortion.

\section{Conclusion}

In this paper, we have explored LHRD's beneficial factors, software environments, and developmental considerations for visualizing high-fidelity VTMS terrain datasets. LHRDs are a novel platform for both the vehicle simulation and pavement engineering communities with their human-size scale and resolution, and more efficient navigation and interaction methods. LHRD visualization is a feasible multiscale visualization tool to improve users' 
subjective understanding of terrain data and encourage collaborative work among engineers. Our case study results suggest that LHRDs coupled with VTMS can effectively support analysis of actual large-scale terrain profiles.

The value of interactive terrain data exploration though LHRD visualization is becoming more important with the explosive growth of large datasets created by advancing measurement systems and high-performance computer simulations. Beyond highfidelity terrain visualizations, our developmental choices and benefits of LHRDs discussed in this paper can be applied to other visualizations of large-scale, high precision datasets for a variety of engineering problems that involve multiscale user tasks, such as the analysis of vehicle models driving over and responding to terrain and visualization of the finite element analysis results.

\section{Acknowledgment}

This research was partially supported by NSF Grant No. CNS1059398.

\section{References}

[1] Kern, J. V., and Ferris, J. B., 2007, "Development of a 3D Vehicle-Terrain Measurement System Part I: Equipment Setup," Proceedings of the Joint North America, Asia-Pacific ISTVS Conference, Fairbanks, AK.

[2] Li, Q., Yao, M., Yao, X., and Xu, B., 2010, "A Real-Time 3D Scanning System for Pavement Distortion Inspection," Meas. Sci. Technol., 21(1), p. 015702.

[3] Easa, S. M., Strauss, T. R., Hassan, Y., and Souleyrette, R. R., 2002, "ThreeDimensional Transportation Analysis: Planning and Design," J. Transp. Eng., 128, pp. 250-258.

[4] Liang, H., Arangarasan, R., and Theller, L., 2007, "Dynamic Visualization of High Resolution GIS Dataset on Multi-Panel Display Using ArcGIS Engine," Comput. Electron. Agric., 58(2), pp. 174-188.

[5] Ni, T., Schmidt, G. S., Staadt, O. G., Livingston, M. A., Ball, R., and May, R., 2006, "A Survey of Large High-Resolution Display Technologies, Techniques, and Applications," Proceedings of IEEE Virtual Reality Conference, pp. 223-236.

[6] Czerwinski, M., Smith, G., Regan, T., Meyers, B., Robertson, G., and Starkweather, G., 2003, "Toward Characterizing the Productivity Benefits of Very Large Displays," Proceedings of Interact, Vol. 3, pp. 9-16.

[7] Ball, R., North, C., and Bowman, D. A., 2007, "Move to Improve: Promoting Physical Navigation to Increase User Performance With Large Displays," Proceedings of the SIGCHI Conference on Human Factors in Computing Systems, San Jose, CA, pp. 191-200.

[8] Ball, R., and North, C., 2007, "Visual Analytics: Realizing Embodied Interaction for Visual Analytics Through Large Displays," Comput. Graph., 13, pp. 380-400.

[9] Smith, H., 2009, "Improving the Quality of Terrain Measurement," M.S. thesis, Mechanical Engineering, Virginia Tech, Blacksburg, VA.

[10] Herr, W. J., 1996, "Highway Profile Measuring System," U.S. Patent No. $5,510,889$.

[11] Ferris, J. B., 1999, "Factors Affecting Perceptions of Ride Quality in Automobiles," Proceedings of ASME Dynamic Systems and Control Division (DSC), 67, pp. 649-654.

[12] Ferris, J. B., and Larsen, J. L., 2002, "Establishing Chassis Reliability Testing Targets Based on Road Roughness," Int. J. Mater. Prod. Technol., 17(5), pp. $453-461$.

[13] Kerchman, V., 2008, "Tire-Suspension-Chassis Dynamics in Rolling Over Obstacles for Ride and Harshness Analysis," Tire Sci. Technol., 36, pp. 158-191.

[14] Pieper, G., Leigh, J., Renambot, L., Verlo, A., Long, L., Brown, M., Sandin, D., Vishwanath, V., Kooima, R., Girado, J., Jeong, B., DeFanti, T., Liu, Q., Katz, M., Papadopoulos, P., Keefe, J., Hidley, G., Dawe, G., Kaufman, I., Glogowski,
B., and Doerr, K., 2009, "Visualizing Science: The OptIPuter Project," SciDAC Review, 12, pp. 32-41.

[15] Yost, B., Haciahmetoglu, Y., and North, C., 2007, "Beyond Visual Acuity: The Perceptual Scalability of Information Visualizations for Large Displays," Proceedings of the SIGCHI Conference on Human Factors in Computing Systems, pp. 101-110.

[16] Schaeffer., B., 2000, “A Software System for Inexpensive VR Via Graphics Clusters," http://isl.uiuc.edu/Publications/dgdpaper.pdf

[17] Schroeder, W. J., Avila, L. S., and Hoffman, W., 2000, "Visualizing With VTK: A Tutorial," IEEE Comput. Graphics Appl., 20, pp. 20-27.

[18] Ball, R., North, C., and Bowman, D. A., 2007, "Move to Improve: Promoting Physical Navigation to Increase User Performance With Large Displays," Proceedings of the SIGCHI Conference on Human Factors in Computing Systems, pp. 191-200.

[19] Tan, D. S., Czerwinski, M., and Robertson, G., 2003, "Women go With the (Optical) Flow," Proceedings of the SIGCHI Conference on Human Factors in Computing Systems, Ft. Lauderdale, FL, pp. 209-215.

[20] Eilemann, S., Makhinya, M., and Pajarola, R., 2008, "Equalizer: A Scalable Parallel Rendering Framework," IEEE Trans. Vis. Comput. Graph., 15, pp. 436-452.

[21] Chen, H., Chen, Y., Finkelstein, A., Funkhouser, T., Li, K., Liu, Z., Samanta, R., and Wallace, G., 2001, "Data Distribution Strategies for High-Resolution Displays," Comput. Graphics, 25(5), pp. 811-818.

[22] Staadt, O. G., Walker, J., Nuber, C., and Hamann, B., 2003 "A Survey and Performance Analysis of Software Platforms for Interactive Cluster-Based MultiScreen Rendering," Proceedings of the Eurographics Workshop on Virtual Environments 2003, pp. 261-270.

[23] Molnar, S., Cox, M., Ellsworth, D., and Fuchs, H., 1994, “A Sorting Classification of Parallel Rendering," IEEE Comput. Graphics Appl., 14(4), pp. 23-32.

[24] Samanta, R., Zheng, J., Funkhouser, T., Li, K., and Singh, J. P., 1999, "Load Balancing for Multi-Projector Rendering Systems," Proceedings of the ACM SIGGRAPH/EUROGRAPHICS Workshop on Graphics Hardware, pp. $107-116$.

[25] Raffin, B., and Soares, L., 2006, "PC Clusters for Virtual Reality," Proceedings of IEEE Virtual Reality Conference, pp. 215-222.

[26] Moreland, K., Wylie, B., and Pavlakos, C., 2001, "Sort-Last Parallel Rendering for Viewing Extremely Large Data Sets on Tile Displays," Proceedings of the IEEE Symposium on Parallel and Large-Data Visualization and Graphics, San Diego, CA, pp. 85-92.

[27] Eilemann, S., 2009, "Equalizer 0.9 Programming and User Guide," http:// www.equalizergraphics.com/documentation.html

[28] Eyescale, 2009, "White Paper: Two Methods for Driving OpenGL Display Walls," http://www.equalizergraphics.com/documents/WhitePapers/ Chromium_Equalizer.pdf

[29] Sandstrom, T. A., Henze, C., and Levit, C., 2003, “The Hyperwall," Proceedings of Coordinated and Multiple Views in Exploratory Visualization, pp. 124-133.

[30] Chi, E. H., Barry, P., Riedl, J., and Konstan, J., 1997, “A Spreadsheet Approach to Information Visualization," Proceedings of IEEE Symposium on Information Visualization, pp. 17-24.

[31] Georgopoulosa, A., Loizosb, A., and Floudac, A., 1995, "Digital Image Processing as a Tool for Pavement Distress Evaluation," ISPRS J. Photogramm. Remote Sens., 50, pp. 23-33.

[32] Shanin, M. Y., 1994, Pavement Management for Airport, Roads, and Parking Lots, Chapman and Hall, New York.

[33] Tan, D. S., and Czerwinski, M., 2003, "Effects of Visual Separation and Physical Discontinuities When Distributing Information Across Multiple Displays," Proceedings of OZCHI, pp. 184-191.

[34] Bi, X., Bae, S.-H., and Balakrishnan, R., 2010, "Effects of Interior Bezels of Tiled-Monitor Large Displays on Visual Search, Tunnel Steering, and Target Selection," Proceedings of the 28th International Conference on Human Factors in Computing Systems, Atlanta, GA.

[35] Robertson, G., Czerwinski, M., Baudisch, P., Meyers, B., Robbins, D., Smith, G., and Tan, D., 2005, "The Large-Display User Experience," IEEE Comput. Graphics Appl., 25, pp. 44-51. 